\title{
Spin injection from Co2MnGa into an InGaAs quantum well
}

Hickey, M. C.; Damsgaard, Christian Danvad; Holmes, S. N.; Farrer, I.; Jones, G. A. C; Ritchie, D. A.; Jacobsen, Claus Schelde; Hansen, Jørn Bindslev; Pepper, M.

Published in:

Applied Physics Letters

Link to article, DOI:

$10.1063 / 1.2938418$

Publication date:

2008

Document Version

Publisher's PDF, also known as Version of record

Link back to DTU Orbit

Citation $(A P A)$ :

Hickey, M. C., Damsgaard, C. D., Holmes, S. N., Farrer, I., Jones, G. A. C., Ritchie, D. A., Jacobsen, C. S., Hansen, J. B., \& Pepper, M. (2008). Spin injection from Co2MnGa into an InGaAs quantum well. Applied Physics Letters, 92(23), 232101. https://doi.org/10.1063/1.2938418

\section{General rights}

Copyright and moral rights for the publications made accessible in the public portal are retained by the authors and/or other copyright owners and it is a condition of accessing publications that users recognise and abide by the legal requirements associated with these rights.

- Users may download and print one copy of any publication from the public portal for the purpose of private study or research.

- You may not further distribute the material or use it for any profit-making activity or commercial gain

- You may freely distribute the URL identifying the publication in the public portal 


\title{
Spin injection from $\mathrm{CO}_{2} \mathrm{MnGa}$ into an InGaAs quantum well
}

\author{
M. C. Hickey, ${ }_{1}^{1, a)}$ C. D. Damsgaard, ${ }^{2}$ S. N. Holmes, ${ }^{1, b)}$ I. Farrer, ${ }^{1}$ G. A. C. Jones, ${ }^{1}$ \\ D. A. Ritchie, ${ }^{1}$ C. S. Jacobsen, ${ }^{2}$ J. B. Hansen, ${ }^{2}$ and M. Pepper ${ }^{1}$ \\ ${ }^{1}$ Cavendish Laboratory, University of Cambridge, J.J. Thomson Avenue, Cambridge CB3 OHE, \\ United Kingdom \\ ${ }^{2}$ Department of Physics, Technical University of Denmark, DK-2800 Lyngby, Denmark
}

(Received 6 January 2008; accepted 11 May 2008; published online 9 June 2008)

\begin{abstract}
We have demonstrated spin injection from a full Heusler alloy $\mathrm{Co}_{2} \mathrm{MnGa}$ thin film into a (100) InGaAs quantum well in a semiconductor light-emitting diode structure at a temperature of $5 \mathrm{~K}$. The detection is performed in the oblique Hanle geometry, allowing quantification of the effective spin lifetime and spin detection efficiency $(22 \pm 4 \%)$. This work builds on existing studies on off-stoichiometric Heusler injectors into similar light-emitting-diode structures. The role of injector stoichiometry can therefore be quantitatively assessed with the result that the spin injection efficiency increases by a factor of approximately 2 as compared with an off-stoichiometric $\mathrm{Co}_{2.4} \mathrm{Mn}_{1.6} \mathrm{Ga}$ injector. (C) 2008 American Institute of Physics. [DOI: 10.1063/1.2938418]
\end{abstract}

Spin injection into degenerate semiconductors such as GaAs is an area of massive interest to the field of spintronics. ${ }^{1}$ The technological application of spin injection are myriad and include the manipulation of classical information carried by spin, initialization, and readout of a spin qubit $^{2}$ and coherent manipulation of spin in the proposed spin field effect transistor. ${ }^{3}$ Single element ferromagnetic transition metals such as Fe are attractive spin injectors as they possess a high Curie temperature and exhibit well understood thin film magnetism. However, the $s-d$ hybridized nature of the band structures of these metals, means that the the Fermi level polarization is limited to the range of $30 \%-$ $40 \%$. There has been a lot of work recently done on understanding the spintronic properties of systems which potentially have only majority spin electrons at the Fermi level, the so-called half metals. In spite of this, there have been very few experimental device implementations of this type of material.

Here, we focus on the Heusler alloy $\mathrm{Co}_{2} \mathrm{MnGa}$ which crystallizes in the $L 2_{1}$ phase. This alloy falls into the general class of Co-based materials which have the the formula $\mathrm{Co}_{2}$ $Y Z$, where the $Y$ and $Z$ atoms are a transition metal and an $s p$ element, respectively. The lattice matching of $\mathrm{Co}_{2} \mathrm{MnGa}$ to GaAs is attractive $(\sim 2 \%)$ and they have Curie temperatures above room temperature. Recent studies of $\mathrm{Co}_{2} \mathrm{MnGa}$ on GaAs(100) (Ref. 4) demonstrate the feasibility of this material system. A fully spin polarized metallic contact may be used as a spin injector without the requirement of a tunnel barrier as it would be immune to the conductivity mismatch problem. ${ }^{5}$

Electronic structure calculations on $\mathrm{Co}_{2} \mathrm{MnGa}$ (Ref. 6) in the framework of density functional theory, indicate a bulk spin polarization in the density of states of $81 \%$ with a total spin moment of $4 \mu_{B}$ per formula. However, the ternary nature of these alloys introduces another level of structural

\footnotetext{
${ }^{a)}$ Present address: The Francis Bitter Magnet Laboratory, M. I. T., $150 \mathrm{Al}-$ bany Street, Cambridge, 02139, USA. Electronic mail: hickey@mit.edu or phymch@leeds.ac.uk.

b) Also at: Toshiba Research Europe Limited, Cambridge Research Laboratory, 208 Cambridge Science Park, Milton Road, Cambridge CB4 0GZ, United Kingdom.
}

complexity as the formation of antisite defects follow Boltzmann statistics and have been shown to detrimentally affect the bulk and interface spin polarization ${ }^{7}$ in $\mathrm{Co}_{2} \mathrm{MnSi}$. In those calculations, the Fermi level spin polarization in the density of states was reduced to about $55 \%$ due to Co antisite defects.

In this paper, we have incorporated a $\mathrm{Co}_{2} \mathrm{MnGa}$ injector into a spin light-emitting diode (LED) structure and investigate spin injection into an $\operatorname{In}_{0.2} \mathrm{Ga}_{0.8}$ As quantum well (QW). This study can be compared with previous spin injection experiments on off-stoichiometric $\mathrm{Co}_{1.6} \mathrm{Mn}_{2.4} \mathrm{Ga}$ LED structures, where spin polarization has been measured to be $13 \%,{ }^{8}$ while $27 \%$ spin injection has been achieved from $\mathrm{Co}_{2} \mathrm{MnGe}$ (Ref. 9) into GaAs. This type of spin LED design is based on a $n-\mathrm{Al}_{0.33} \mathrm{Ga}_{0.67} \mathrm{As}$ Schottky LED structures which were used to successfully demonstrate spin injection from $\mathrm{Fe}$ into GaAs/AlGaAs QWs. ${ }^{10}$ Further, the oblique Hanle geometry can be used to estimate the effective spin lifetime in the QW. ${ }^{11}$

The structure comprises a GaAs based LED structure with a surface Schottky barrier onto which a ferromagnetic metal layer is grown. The III-V wafer is grown by standard molecular beam epitaxy techniques and is in situ transferred under UHV conditions into a magnetic metals chamber where a $10 \mathrm{~nm} \mathrm{Co} \mathrm{Cn}_{2} \mathrm{Mna}$ layer is grown at $250{ }^{\circ} \mathrm{C}$ with a deposition rate of $3 \AA \mathrm{min}^{-1}$ followed by a $3 \mathrm{~nm} \mathrm{Au}$ cap.

The III-V part of the heterostructure consists of the following layers: $15 \mathrm{~nm} n$ - $\mathrm{Al}_{0.33} \mathrm{Ga}_{0.67} \mathrm{As}\left(3.3 \times 10^{18} \mathrm{~cm}^{-3}\right) /$ $15 \mathrm{~nm} \quad n-\mathrm{Al}_{0.33} \mathrm{Ga}_{0.67} \mathrm{As} \quad\left(1 \times 10^{18} \mathrm{~cm}^{-3}\right) / 50 \mathrm{~nm} \quad n$-GaAs $\left(1 \times 10^{18} \mathrm{~cm}^{-3}\right) / 50 \mathrm{~nm} \quad$ GaAs$/ 5 \mathrm{~nm} \quad \operatorname{In}_{0.2} \mathrm{Ga}_{0.8} \mathrm{As} / 100 \mathrm{~nm}$ $\mathrm{GaAs} / 10 \mathrm{~nm} \quad \operatorname{In}_{0.2} \mathrm{Ga}_{0.8} \mathrm{As} / 100 \mathrm{~nm} \quad \mathrm{In}_{0.2} \mathrm{GaAs} / 500 \mathrm{~nm}$ $p$-GaAs $\left(1 \times 10^{18} \mathrm{~cm}^{-3}\right)$ on a $(100) p$-GaAs substrate. This device wafer was designed to have two QWs in order to observe optical recombination from two distinct regions in the semiconductor. However, only recombination from one (5 nm) QW is observed. The metal/semiconductor heterostructure (spin LED) was then processed using $\mathrm{Ar}^{+}$ion etching techniques to make a $240 \mu \mathrm{m} \mathrm{Co}_{2} \mathrm{MnGa}$ mesa inside a larger $500 \mu \mathrm{m}$ mesa of the semiconductor part of the device heterostructure. The device band diagram, electroluminescence and current-voltage characteristics are plotted in Fig. 1. 

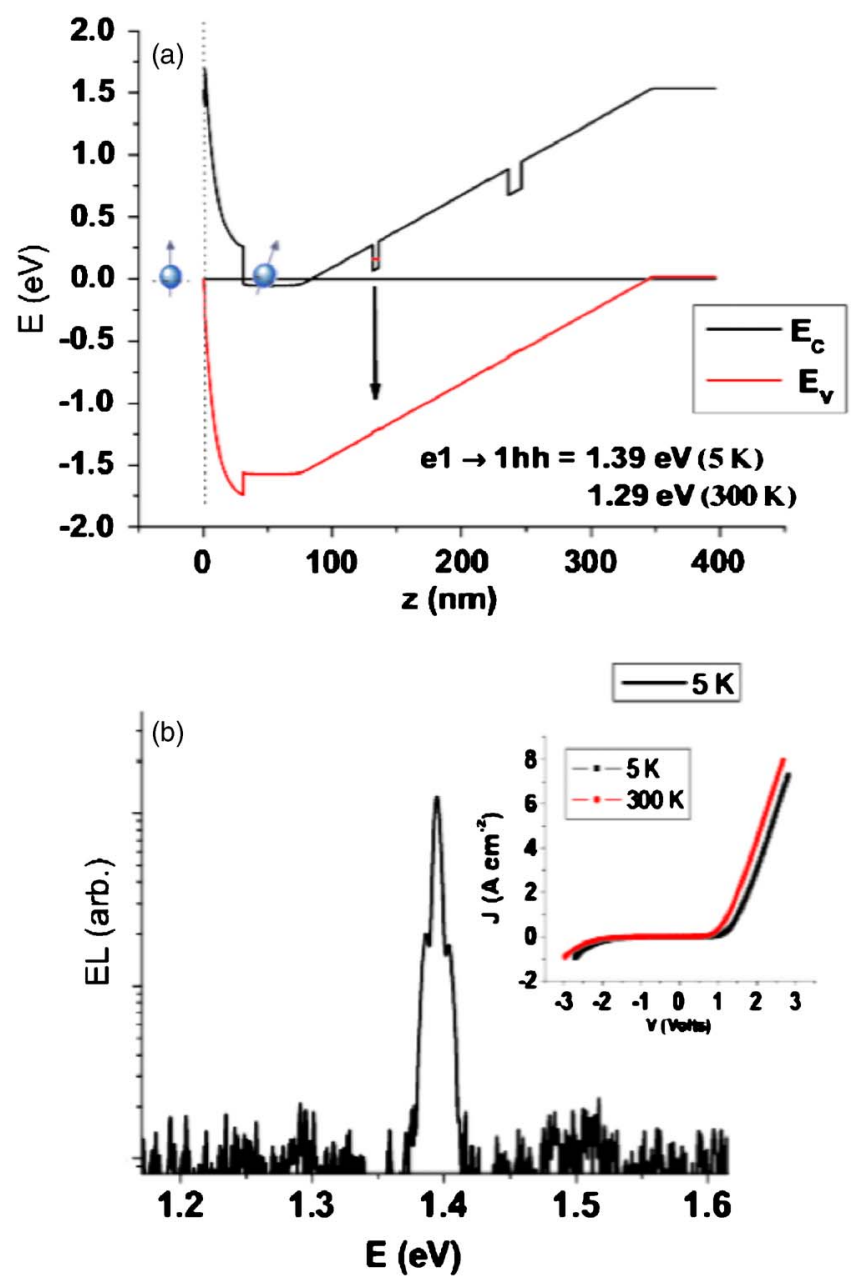

FIG. 1. (Color online) (a) The self-consistent field calculated band diagram of the spin LED structure, showing the $1 e \rightarrow 1 h h$ excitonic transition. (b) The electroluminescence spectrum at $5 \mathrm{~K}$ confirming that the emission is dominated by the $1 e \rightarrow 1 h h$ free exciton.

The injected spin polarization is detected via a measurement of the circular polarization of the emitted light from the recombination region at $5 \mathrm{~K}$. This method has become a standard technique in devices of this type (spin LEDs) and involves an oscillating quarter wave retarder and a static linear polarizer as described elsewhere. ${ }^{10-12}$ The polarization of the injected electrons is related to the optical polarization via the relation,

$$
P=\frac{I_{\sigma+}-I_{\sigma_{-}}}{I_{\sigma+}+I_{\sigma_{-}}} \propto \frac{n_{\uparrow}-n_{\downarrow}}{n_{\uparrow}+n_{\downarrow}},
$$

where $I_{\sigma_{ \pm}}$is the intensity of right or left circularly polarized light and $n_{\uparrow, \downarrow}$ is the density of spin up or down electrons which undergo recombination. In the experimental configuration here, the magnetic field is applied at $30^{\circ}$ to the plane of the thin film contact and the emitted EL is collected along the (100) crystallographic direction (the growth axis) and normal to the plane of the thin film.

The optical polarization as a function of applied magnetic field $[P(B)]$ is plotted in Fig. 2, having subtracted a small negative linear component, arising from a weak Zeeman splitting $g \mu_{B} B \ll k T$. $P(B)$ is proportional to the $S_{z}$ component of the spin and the total spin state $\vec{S}$, obeys the Bloch equation ${ }^{13}$ for the time evolution of spin states. The injected spins undergo Larmor precession and the radius of precesDownloaded 21 Jun 2010 to 192.38.67.112. Redistribution subject

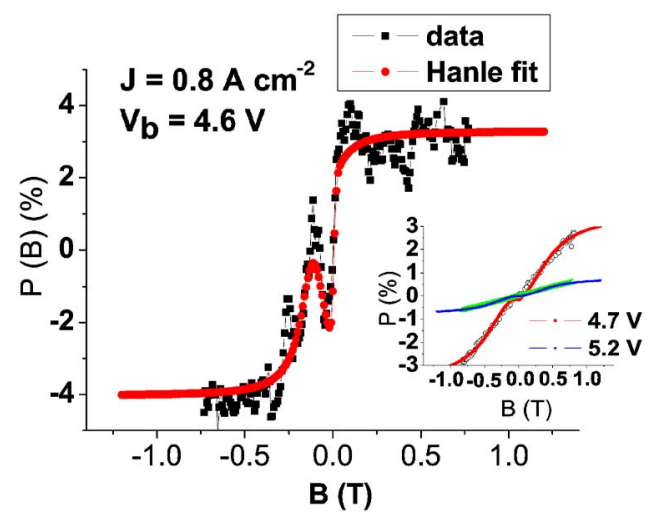

FIG. 2. (Color online) Oblique Hanle effect in the spin LED at $T=5 \mathrm{~K}$ at a bias voltage $4.6 \mathrm{~V}$. The effective spin lifetimes $\left(T_{s}^{*}\right)$ are estimated from fits to Bloch theory. The inset shows Hanle effect measurements at higher bias voltages $\left(\mathrm{V}_{b}\right)$ at $5 \mathrm{~K}$, showing a decrease in amplitude accompanied by a widening of the curves.

sion is determined by the spin scattering rate. These processes underpin the oblique Hanle effect. The steady-state Bloch solution can be written as follows:

$$
S_{z}=\frac{T_{s}}{\tau} \frac{S_{0 z}+S_{0 y} T_{s}^{2} \Omega_{y} \Omega_{z}+S_{0 z} T_{s}^{2} \Omega_{z}^{2}}{1+T_{s}^{2} \Omega_{y}^{2}+T_{s}^{2} \Omega_{z}^{2}},
$$

$\boldsymbol{\Omega}=\left(\Omega_{x}, \Omega_{y}, \Omega_{z}\right)=g \mu_{B} \mathbf{B} / \hbar$ is the Larmor frequency arising from the oblique magnetic field $\mathbf{B}=\left(0, B_{y}, B_{z}\right), g$ and $\mu_{B}$ are the Landé $g$ factor and the Bohr magnetron, respectively. $\mathbf{S}_{0}$ is the initial spin state which is directly related to the orientation of magnetization in the injecting film. Thin film anisotropy ensures that the $S_{0 z}$ terms are small compared to the $S_{0 y}$ term as the film magnetization tilts by about $10^{\circ}$ out of plane in this geometry. The relevant timescales in the experiment are $T_{s}^{*}$ and $\tau$, which are the effective spin lifetime, and the radiative lifetime respectively. The spin polarization of the injected electron density $(\Pi)$ is related to the optical polarization $(P)$ via $\Pi T_{s}^{*} / \tau=P /(\cos \phi \sin \phi)$, where $\phi\left(=30^{\circ}\right)$ is the angle between the oblique magnetic field and the plane of the $\mathrm{Co}_{2} \mathrm{MnGa}$ film.

The Hanle effect data at $5 \mathrm{~K}$ and fits to Bloch theory shown in Fig. 2 give effective spin lifetimes of $T_{s}^{*}$ $=(160 \pm 30)$ ps at $V_{b}=4.76 \mathrm{~V}$, based on a $g$ factor of $|g|=$ -0.8 taken from data on $\operatorname{In}_{0.21} \mathrm{Ga}_{79} \mathrm{As} / \mathrm{GaAs}$ QWs (Ref. 14) and a measured radiative lifetime of $\tau=400 \mathrm{ps}$ by photoluminescence. The kink in the curve around zero field is associated with a nuclear dipole shift. This fit gives a value of $\Pi=22 \% \pm 4 \%$ for the injected spin polarization, as detected in the QW. The bias dependent spin injection is shown in Fig. 3. At $V_{b}<4.76 \mathrm{~V}$, the light intensity drops and the polarization data become unreliable as evidenced by the error bars on the plot. At higher bias, there is a reduction in observed spin polarization in the QW accompanied by an increase in the Hanle half-width. This corresponds to a shorter $T_{s}^{*}$. This is due to the enhanced electric field which the spins see in the $5 \mathrm{~nm}$ InGaAs QW region. The electric field gives rise to a more pronounced spin orbital field from the asymmetrizing of the confinement potential. Toward the higher bias limit, the optical polarization drops to a constant $P$ $\sim 1 \%$. The drop in measured polarization is due to the decrease in $T_{S}^{*}$, and hence a decrease in the spin detection efficiency $\left(\eta=T_{S}^{*} / \tau\right)$. This decrease in $T_{S}^{*}$ is evidenced by the increase the Hanle half-width in the $P(B)$ curved in Fig. 2. 


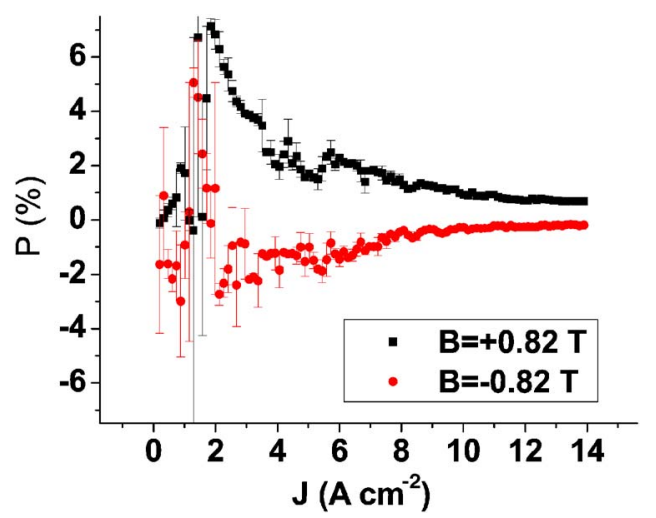

FIG. 3. (Color online) The bias current dependent polarization at $5 \mathrm{~K}$ showing the change in sign due to magnetic field reversal. The error bars arise at low bias due to the drop in light intensity.

We have demonstrated spin injection from the Heusler alloy $\mathrm{Co}_{2} \mathrm{MnGa}$. The maximum optically measured spin polarization was found to be $4.0 \% \pm 0.3 \%$ in the low bias regime. The value of electron spin polarization inferred from this measurement in the Hanle geometry was found to be $\Pi=22 \% \pm 4 \%$, having established an effective spin lifetime of $T_{s}^{*}=160 \pm 30$ ps. Despite the predictions of a high degree of spin polarization in the injecting metal and the optimized Schottky barrier configuration, the injected spin polarization does not improve on that previously achieved with $\mathrm{Fe}$. Comparing this result with off-stoichiometric $\mathrm{Co}_{2.4} \mathrm{Mn}_{1.6} \mathrm{Ga}$, we find that there is an increase in the injected spin polarization by a factor of 2 .
M. C. Hickey would like to acknowledge financial support from the Cambridge European Trust, Toshiba Research Europe Limited, and the Engineering and Physical Sciences Research Council.

${ }^{1}$ D. D. Awschalom, D. Loss, and N. Samarth, Semiconductor Spintronics and Quantum Computation (Springer Nanoscience and Technology, Berlin, Germany, 2002).

${ }^{2}$ D. Loss and D. P. DiVincenzo, Phys. Rev. A 57, 120 (1998).

${ }^{3}$ J. Schliemann, J. C. Egues, and D. Loss, Phys. Rev. Lett. 90, 146801 (2003).

${ }^{4}$ D. Heiman, Y. Chen, D. Basiaga, L. Zhang, and P. G. Murugesan, Compound Semiconductors Proceedings 184, 153 (2005).

${ }^{5}$ G. Schmidt, D. Ferrand, L. W. Molenkamp, A. T. Filip, and B. J. van Wees, Phys. Rev. B 62, R4790 (2000).

${ }^{6}$ M. Sargolzaei, M. Richter, K. Koepernik, I. Opahle, H. Eschrig, and I. Chaplygin, Phys. Rev. B 74, 224410 (2006).

${ }^{7}$ S. Picozzi, A. Continenza, and A. J. Freeman, Phys. Rev. B 69, 094423 (2004).

${ }^{8}$ M. C. Hickey, C. D. Damsgaard, I. Farrer, S. N. Holmes, A. Husmann, J. B. Hansen, C. S. Jacobsen, D. A. Ritchie, R. F. Lee, G. A. C. Jones, and M. Pepper, Appl. Phys. Lett. 86, 252106 (2005).

${ }^{9}$ X. Y. Dong, C. Adelmann, J. Q. Xie, C. J. Palmstrom, X. Lou, J. Strand, P. A. Crowell, J.-P. Barnes, and A. K. Petford-Long, Appl. Phys. Lett. 86, 102107 (2005).

${ }^{10}$ A. T. Hanbicki, B. T. Jonker, G. Itskos, G. Kioseoglou, and A. Petrou, Appl. Phys. Lett. 80, 1240 (2002).

${ }^{11}$ V. F. Motsnyi, P. V. Dorpe, W. V. Roy, E. Goovaerts, V. I. Safarov, G. Borghs, and J. D. Boeck, Phys. Rev. B 68, 245319 (2003).

${ }^{12}$ R. Fiederling, M. Keim, G. Reuscher, W. Ossau, G. Schmidt, A. Waag, and L. W. Molenkamp, Nature (London) 402, 787 (1999).

${ }^{13}$ F. Bloch, Phys. Rev. B 70, 460 (1946).

${ }^{14}$ B. Kowalski, V. Zwiller, W. Wiggren, P. Varekamp, M. Miller, M. Pistol, P. Omling, and L. Samuelson, Jpn. J. Appl. Phys., Part 1 37, 245319 (1998). 